\section{Prevenção de infecção pelo HIV por intermédio da utilização do grupo operativo entre homens que fazem sexo com homens, São Paulo, Brasil}

\author{
HIV prevention using the operative group \\ approach among men who have sex \\ with men in São Paulo, Brazil
}

\footnotetext{
${ }^{1}$ Instituto de Psicologia, Universidade de São Paulo, São Paulo, Brasil.

2 Faculdade de Saúde Pública, Universidade de São Paulo, São Paulo, Brasil. 3 Secretaria do Verde e do Meio Ambiente, Prefeitura Municipal de São Paulo, São Paulo, Brasil. 4 University of California, San Francisco, U.S.A.

Correspondência R. Colosio Núcleo de Estudos para a Prevenção da AIDS/

Laboratório de Estudos em Psicanálise e Psicologia Social, Instituto de Psicologia, Universidade de São Paulo.

Av. Prof. Mello Moraes 1721 Bloco A, São Paulo, SP 05508-030, Brasil. robcol@usp.br
}

\begin{abstract}
This study aimed to evaluate the operative group as a preventive approach among men who have sex with men that use two public health services in the city of São Paulo, Brazil. One hundred volunteers were randomly allocated to two groups (intervention and control, with 50 each). All participants answered questionnaires in two phases: before the intervention and six months after its conclusion. Effect was measured by comparing the groups for the following outcomes: median number of anal sex acts without condoms and responses from the participants to questions about HIV infection. 69 participants completed the study (34 in the prevention group and 35 in the control group). Analysis showed a decrease in the number of unprotected anal sex acts ( $p=$ 0.029 ) and an increase in the number of answers favoring prevention in the intervention group. The results indicate that the study group was responsive to a safer sex operative group intervention. Further research is necessary to evaluate the feasibility of this prevention approach as a public health strategy, including other social groups.
\end{abstract}

HIV; Acquired Immunodeficiency Syndrome; Male Homosexuality; Disease Prevention; Social Psychology

\author{
Robson Colosio 1 \\ Maria Inês Assumpção Fernandes 1 \\ Denise Pimentel Bergamaschi 2 \\ Ianni Régia Scarcelli 1 \\ Isabel Cristina Lopes ${ }^{3}$ \\ Norman Hearst 4
}

\section{Introdução}

A história da epidemia de AIDS no Brasil e, de modo semelhante, no Município de São Paulo, vem apresentando uma importante modificação no que concerne ao número de casos notificados de AIDS entre homens que fazem sexo com homens (HSH). Os dados apontam um decréscimo acentuado na categoria de exposição homossexual, comparada às demais categorias: de 54,5\% em 1982 para 16,3\% em 2002, em nível nacional 1, e de $66,7 \%$ em 1990 para 29,3\% em 2001, na cidade de São Paulo ${ }^{2}$. Apesar dessa importante mudança, a população de HSH mantém um quadro expressivo de infecção pelo HIV, aproximadamente um quarto do total de casos, motivo pelo qual é apontada como grupo preferencial para ações constantes de prevenção, a fim de que aumente a prevalência da prática de sexo mais seguro. Essa diretriz releva-se pelos dados colhidos entre usuários de Centros de Testagem e Aconselhamento (CTA), na cidade de São Paulo, com resultado positivo para o HIV, os quais mostram um percentual elevado de pessoas que não adotam o uso de preservativo em todas as relações sexuais; apenas $35 \%$ dos homens relatam seu uso sistemático. Do total da amostra, $49 \%$ denominaram-se homo-bissexuais 3 .

Autores como Paiva 4 , Parker 5 e Terto Jr. 6 chamam a atenção para que programas de prevenção considerem a importância da dimensão sócio-cultural como fator importante da elabo- 
ração de estratégias para diminuição do grau de vulnerabilidade ao HIV, em particular para a população de HSH. Destacam também a revisão da adoção de modelos comportamentais que visem apenas à mudança de conduta pela normatização da prática contínua de sexo mais seguro. Essa perspectiva de ação apóia-se, entre outras pesquisas, naquela realizada por Díaz 7 , em seu estudo sobre homossexuais latinos de San Francisco, Estados Unidos, que revela como a epidemia de AIDS os têm afetado de forma elevada e desproporcional, quando comparados a outros subgrupos homossexuais. Este autor relaciona esse fenômeno à ação de complexos fatores sócio-culturais que se expressam pelo machismo, homofobia, silêncio sobre questões sexuais, ruptura familiar, pobreza e racismo. Tais fatores atuam diretamente nas atitudes de auto-regulação dessa população em face de situações de risco, cujos desdobramentos resultam em baixa auto-estima, fatalismo sobre a inevitabilidade de infecção pelo HIV, percepção de baixo controle sexual e isolamento social.

Em virtude dessas questões de ordens tão diversas e complexas, inerentes ao campo social, considerou-se como estratégia necessária à prevenção o trabalho em grupo, por considerá-lo um espaço de produção simbólica, no qual as afetividades se expressam e as relações se concretizam, seja na dimensão psíquica interna (intrapsíquica), seja na relação com o outro (intersubjetiva), seja no que se herda psiquicamente da família e da cultura (trans-subjetiva), segundo Fernandes ${ }^{8}$. Esse recorte teórico fundamenta-se na psicanálise freudiana ${ }^{9} \mathrm{e}$, portanto, pressupõe sua hipótese fundamental e constitutiva: o inconsciente - dimensão psíquica, diferenciada do consciente, que possui conteúdos, mecanismos e energia específicos, os quais só se tornam acessíveis à consciência depois de superados processos de resistência.

Dessa teoria, desdobra-se uma concepção de sujeito, elaborada por Pichon-Rivière 10, que o considera como emergente do processo dialético e interacional grupal, no qual, ao mesmo tempo, o sujeito atua como determinante (do processo) e determinado (pelo processo). A interação grupal dá-se, especialmente, pela linguagem, na troca comunicacional de representações (pessoais e sociais), as quais se formam e se articulam em nível tanto inconsciente (na forma de fantasias, angústias, medos não identificáveis etc.), quanto consciente (idéias, preconceitos, atitudes, discursos etc.). Desse modo, grupo é o lugar privilegiado para investigar e intervir sobre as representações que, enquanto partes constitutivas da interação grupal, estão articuladas e determinadas pelos entrecruzamentos de diversas ordens (social, cultural, econômica, psíquica, histórica etc.). Por todas estas condições, o trabalho em grupo possibilita promover ações em prevenção contra a infecção pelo HIV.

Este artigo apresenta os resultados de um estudo experimental que investiga os efeitos da adoção de um procedimento de intervenção em grupo, elaborado por Pichon-Rivière 11 e denominado grupo operativo, no trabalho de prevenção de infecção pelo HIV. O estudo foi dirigido à população de HSH, usuários de dois centros de testagem e aconselhamento da cidade de São Paulo. Para a elaboração da estratégia metodológica da pesquisa, consideraram-se tanto o trabalho de Johnson et al. 12, sobre a escassez de estudos com desenhos padronizados, com controle rigoroso de resultados e tratamento estatístico, quanto a revisão bibliográfica de pesquisas com abordagem psicanalítica em prevenção de AIDS, elaborada por Moreno 13; com o mesmo resultado. Considerou-se, ainda, a importância da promoção de diálogo entre áreas afins ao trabalho de prevenção de AIDS, pela aproximação de abordagens metodológicas. Para esta tarefa, contou-se com o trabalho do grupo de pesquisadores da University of California-San Francisco, Estados Unidos 14

\section{Método}

\section{Campo da pesquisa}

O estudo foi desenvolvido com HSH, usuários de dois CTAs da Prefeitura do Município de São Paulo: Henfil e Santo Amaro, escolhidos dentre os serviços de maior procura por essa população e por apresentarem os maiores índices de testes com resultados positivos dentre os programas de atendimento específico a esse grupo, na cidade de São Paulo 3. Por meio de um questionário de rotina, aplicado pelo serviço, foram identificados usuários da população de HSH e maiores de 18 anos (critérios de elegibilidade), sem considerar seu status sorológico. Foram inicialmente convidados a participar do estudo 147 usuários, os quais, em entrevista individual, receberam explicações sobre os objetivos do estudo e as condições nas quais seria desenvolvido. Aos concordantes, apresentou-se o termo de consentimento informado e fez-se o colhimento de assinaturas, até o preenchimento do número necessário de participantes.

O projeto deste estudo foi submetido ao Comitê de Ética da University of California-San Francisco e ao Centro de Referência do Programa Estadual de DST/AIDS de São Paulo (CRTPE-DST/AIDS). 


\section{Desenho do estudo e materiais}

Entre maio de 2002 a fevereiro de 2003, realizouse um estudo de intervenção controlado, com alocação aleatória de voluntários, na sede do CRT-PE-DST/AIDS, em São Paulo. Inicialmente, foram formados grupos de intervenção e controle (100 voluntários, 50 em cada grupo), distribuídos por meio de sorteio realizado por uma pessoa não envolvida diretamente no recrutamento. Em razão de o número ideal de participantes de grupo operativo ser entre 15 e 20 pessoas, procedeuse à formação de quatro subgrupos: dois grupos de intervenção - A e B; e dois grupos de controle - C e D, com 25 participantes cada. Todos os grupos participaram de sessões do grupo operativo, mas em momentos diferentes. No primeiro mês de trabalho (Fase 1, Mês 1), os participantes do grupo intervenção A e controle $\mathrm{C}$ responderam a um questionário (baseline), pré-testado e desenvolvido especialmente para esta pesquisa. Iniciou-se o processo de intervenção com o grupo A, enquanto o grupo C aguardou até a segunda aplicação questionário. O mesmo processo repetiu-se no mês seguinte (Mês 2) para os grupos de intervenção B e controle D. Seis meses após o início do estudo (Fase 2, follow-up), procedeu-se à segunda aplicação do questionário inicial (avaliação de impacto da intervenção) para os grupos A e C (Mês 7) e para os grupos B e D (Mês 8). Em seguida, por questões éticas, os grupos $\mathrm{C}$ e $\mathrm{D}$, que inicialmente serviram como controle, foram submetidos à mesma intervenção dos grupos $\mathrm{A}$ e $\mathrm{B}$, porém não foram considerados na análise final dos dados.

O desenho utilizado neste estudo segue uma estratégia metodológica freqüentemente utilizada em epidemiologia - ensaio clínico aleatorizado, com grupo de controle-, descrita como a que melhor permite avaliar o efeito de uma intervenção ${ }^{14}$, pois, além de adotar grupo de controle, que possibilita realizar comparações, permite, pela alocação aleatória, controlar as diferenças entre os grupos de intervenção e de controle, igualando-os sob todos os aspectos, com exceção da variável intervenção. Para o cálculo do tamanho da amostra, considerou-se como hipótese nula a não-existência de diferença entre relatos de exposição nos grupos; nível de significância de $5 \%$; poder do teste de $80 \%$ e proporções de $80 \%$ e $50 \%$ de relatos de sexo desprotegido nos grupos de controle e de intervenção. Obteve-se, assim, o número de 38 indivíduos em cada grupo que, após a consideração de perdas $(24 \%$, sendo $10 \%$ para possíveis perdas e recusas e $14 \%$ para manter o poder do estudo, após controle de fatores de confusão), resultou em 50 indivíduos para cada grupo (conforme tabela 13b de Designing
Clinical Research: An Epidemiologic Approach 14). O esquema de desenvolvimento do estudo está resumido na Figura 1.

O questionário adotado baseou-se no modelo de redução de risco de AIDS (AIDS Risk Reduction Model) 15. Nele, incluiu-se uma seção investigativa sobre características sócio-demográficas, práticas sexuais e uso de preservativo referentes a cada relação sexual no período de 30 dias anteriores à sua aplicação. Incluíram-se, também, perguntas sobre sexo seguro, crenças e conhecimentos relativos a HIV e AIDS, em forma de sentenças com cinco respostas possíveis: discordo totalmente, discordo em parte, indiferente, concordo em parte e discordo totalmente, segundo escala de Likert 16.

\section{Intervenção: grupo operativo}

O grupo operativo desenvolveu-se em cinco sessões semanais de uma hora e meia. O trabalho foi conduzido por dois coordenadores, psicólogos especializados neste procedimento de intervenção e um observador (anotações para posterior trabalho de supervisão). Todas as sessões foram registradas em fita cassete e transcritas. Ao início dos trabalhos de grupo, os coordenadores esclareceram dúvidas sobre a pesquisa e apresentaram a tarefa (conceito definido para grupo operativo): discutir sobre as relações entre "proteção e sexualidade".

O trabalho de intervenção desenvolveu-se apoiado no conceito de representação afetiva, tal como formulado por Freud 9, o qual vincula uma energia psíquica e uma idéia, seja esta consciente, seja inconsciente. No que se refere ao inconsciente, trabalha-se com o conceito de recalque, um mecanismo psíquico inconsciente que desloca do consciente para o inconsciente as representações inaceitáveis ao sujeito ou ao seu grupo. Esse processo cria uma cisão entre a energia psíquica e a idéia, mas não elimina a importância das representações, as quais, do plano inconsciente, continuam a determinar ações que, embora sem sentido, mantêm comportamentos não ajustados à realidade (ações de risco).

A condução dos grupos operativos deu-se como descrito por Pichon-Rivière 11 e Fernandes 8 , que orientam o trabalho do coordenador de grupo no sentido de promover a articulação entre pensamento e afeto, pelo apontamento das fantasias inconscientes e a ação dos mecanismos de cisão e enrijecimento no grupo. Esse trabalho tem a perspectiva de criar espaços para o desenvolvimento de articulações associativas, fundamentais para promover processos de ressignificação das representações conscientes envolvidas na decisão de proteger-se da infecção pelo HIV, 


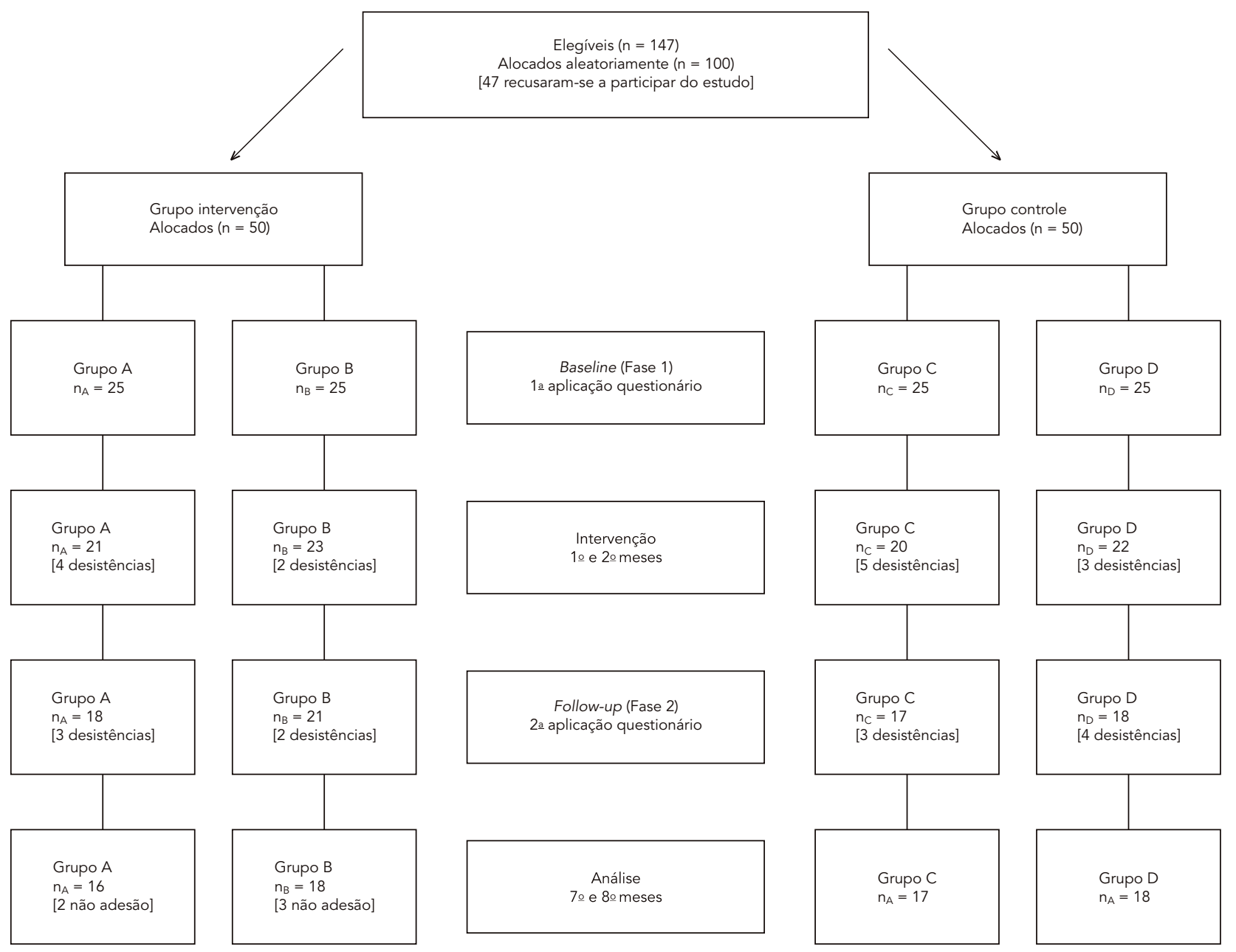

assim como possibilitar a ascensão das representações inconscientes à consciência.

\section{Análise dos dados}

As variáveis sócio-demográficas - idade, local de nascimento, tipo de moradia, número de pessoas com quem divide o quarto, escolaridade e renda per capita - foram utilizadas para caracterização dos grupos estudados. As variáveis sobre prática sexual geraram a variável sexo anal desprotegido, a qual consistia do número de vezes que cada participante tivera uma relação sexual anal sem uso de preservativo. Esse é um índice central na avaliação da parte quantitativa, pois, segundo
Vittnghoff et al. 17, refere-se ao tipo de prática sexual mais eficiente de infecção pelo HIV e em torno da qual centra-se a maioria das pesquisas em prevenção.

Realizou-se a verificação da semelhança entre grupo de controle e de intervenção com relação às variáveis mencionadas anteriormente, utilizando-se teste t de Student, para igualdade de médias para amostras independentes; teste não paramétrico de Mann-Whitney-Wilcoxon, quando a distribuição não era normal, e teste qui-quadrado de Pearson, para verificação de associação.

O estudo das diferenças entre os grupos de controle e de intervenção, no que diz respeito às 
variáveis sobre práticas, crenças e conhecimentos, foi realizado com base na comparação de proporções, por meio do teste exato de Fisher. As mudanças de opinião que refletiam atitudes direcionadas à prevenção foram identificadas e apresentadas na Tabela 1 .

Os números médios de situações de sexo anal sem uso de preservativo foram comparados pela análise de perfis, utilizando-se a técnica de análise de variância (ANOVA), com dois fatores (grupo e fase) e observações repetidas em fase. Realizouse o teste de esfericidade, com cálculo do valor $\varepsilon$ de Box. Os dados foram armazenados em arquivo de dados do Epi Info (Centers for Disease Control and Prevention, Atlanta, Estados Unidos) e processados com auxílio do Stata versão 7 (Stata Corporation, College Station, Estados Unidos), sem utilizar a estratégia de análise por intenção de tratamento. Foi estabelecido como critério de adesão à intervenção o comparecimento a, pelo menos, três sessões de grupo operativo.

\section{Resultados}

\section{Descrição dos indivíduos: comparação entre os grupos}

A comparação dos grupos no baseline, para avaliação da aleatorização, não indicou evidência estatística de diferença entre eles quanto à idade $(\mathrm{p}=0,556)$, tipo de moradia $(\mathrm{p}=0,145)$ e renda $(\mathrm{p}$ $=0,968$ ) (dados não apresentados).

Dos indivíduos que terminaram o estudo, observou-se idade média de 30,6 anos, dos quais $7 \%$ tinham entre 18 e 20 anos e $16 \%$, idade de 40 anos e mais. Grande parte dos participantes nasceu nas regiões Centro-Oeste ou Sudeste do país (81\%). Quanto ao tipo de moradia, a distribuição foi igualmente representada pelas categorias "própria", "alugada" e "mora com parentes". Sobre a escolaridade, observou-se que a maior parte deles (77\%; IC95\%: 65,1-86,1\%) ultrapassou o primeiro grau (8 anos de estudo); destes, 19\% tinham curso superior completo. Em relação à renda per capita, $52 \%$ ganhavam menos de cinco salários mínimos, cada salário equivalendo, na época, a R\$ 140,00. A renda média per capita é de 6,0 salários mínimos (IC95\%: 3,7-8,4) (Tabela 2).

Ao final do estudo, do total de 50 indivíduos em cada grupo, permaneceram $34(68 \%)$ no grupo de intervenção e 35 (70\%) no grupo de controle. A comparação entre os indivíduos que aderiram à intervenção e os que não aderiram não revelou evidências de diferenças estatísticas, com valores de $\mathrm{p}$ iguais para idade $(\mathrm{p}=0,583)$, escolaridade $(\mathrm{p}=0,947)$ e renda $(\mathrm{p}=0,981)($ dados não apresentados).

\section{Características de comportamento após a realização do grupo operativo}

Após a realização do grupo operativo, observouse diferença entre os grupos de controle e de intervenção para questões relacionadas a práticas, crenças e conhecimento sobre transmissão do HIV e uso de preservativo. Observa-se, na Tabela 1 , que $28 \%$ dos participantes do grupo de intervenção mudaram sua opinião quanto a "não gostar da camisinha" e de "estar cansado de fazer sexo protegido"; nenhum do grupo de controle mudou sua opinião. No que se refere a conhecimentos, observa-se que grande parte dos participantes do grupo de intervenção $(76 \%)$ e menos da metade do grupo de controle (43\%) discordaram da afirmação que "repetidas exposições ao HIV, com o tempo, podem prejudicar o sistema imunológico do portador", quando se esperava que concordassem com ela. Foram também observados altos percentuais (84\%) de respostas discordantes de aspectos como "um jeito de evitar o HIV é ter sexo anal com quem tem o mesmo resultado de HIV que você" (Tabela 1 ).

\section{Utilização de preservativo}

Observou-se diminuição do número médio de ocasiões com prática de sexo anal desprotegido entre os participantes do grupo de intervenção e aumento deste número médio entre os participantes do grupo de controle na Fase 2 do estudo (Figura 2). Pela ANOVA, pode-se, portanto, observar existência de efeito da intervenção $(\mathrm{p}=$ 0,029) (Figura 2).

\section{Discussão}

Os resultados deste estudo indicam a possibilidade de HSH beneficiarem-se de um programa de prevenção pela utilização do grupo operativo. A verificação desse efeito favorável, mesmo após o intervalo de seis meses, é um achado importante que merece destaque. Esse efeito verificase tanto na avaliação das variáveis sobre prática sexual com (ou sem) uso de camisinha, quanto nas respostas sobre crenças, atitudes e conhecimento sobre infecção pelo HIV.

Esses achados estão em acordo com o que Pichon-Rivière 11 interpreta como um efeito do processo grupal de mudança, pois cada sujeito tem a possibilidade de entrar em contato com "esquemas" dos outros membros do grupo (conceito definido pelo autor como Esquemas Conceituais, Referenciais e Operativos - ECRO). Esse processo permite-lhes acesso, especialmente, a representações, conscientes e inconscientes, so- 
Comparação dos grupos de controle e de intervenção quanto a atitudes e conhecimento.

\begin{tabular}{|c|c|c|c|c|c|}
\hline \multirow[t]{2}{*}{ Atitudes e conhecimento } & \multicolumn{2}{|c|}{ Controle } & \multicolumn{2}{|c|}{ Intervenção } & \multirow{2}{*}{$\begin{array}{c}\text { Valor de p } \\
\text { teste exato } \\
\text { de Fisher }\end{array}$} \\
\hline & $\mathbf{n}$ & $\%$ & $\mathbf{n}$ & $\%$ & \\
\hline \multicolumn{6}{|c|}{ Na última vez em que teve sexo sem camisinha, você não quis usá-la por não gostar } \\
\hline \multicolumn{6}{|l|}{ Atitude de prevenção * } \\
\hline Sim & 5 & 20 & 14 & 50 & $<0,001$ \\
\hline Não & 20 & 80 & 4 & 50 & \\
\hline Total & 25 & 100 & 18 & 100 & \\
\hline \multicolumn{6}{|c|}{ Estar cansado de fazer sexo protegido } \\
\hline \multicolumn{6}{|l|}{ Atitude de prevenção * } \\
\hline Sim & 8 & 32 & 14 & 78 & 0,005 \\
\hline Não & 17 & 68 & 4 & 22 & \\
\hline Total & 25 & 100 & 18 & 100 & \\
\hline \multicolumn{6}{|c|}{ Pensando sobre a camisinha. Quanto você concorda ou discorda das seguintes } \\
\hline \multicolumn{6}{|c|}{ afirmações repetidas exposições ao HIV, com o tempo, podem prejudicar } \\
\hline \multicolumn{6}{|c|}{ o sistema imunológico do portador } \\
\hline \multicolumn{6}{|l|}{ Atitude de prevenção ** } \\
\hline Sim & 20 & 57 & 8 & 24 & 0,007 \\
\hline Não & 15 & 43 & 26 & 76 & \\
\hline Total & 35 & 100 & 34 & 100 & \\
\hline \multicolumn{6}{|c|}{ Um jeito de evitar o HIV é ter sexo anal com quem tem o mesmo } \\
\hline \multicolumn{6}{|l|}{ resultado de HIV que você } \\
\hline \multicolumn{6}{|l|}{ Atitude de prevenção * } \\
\hline Sim & 20 & 57 & 29 & 85 & 0,016 \\
\hline Não & 15 & 43 & 5 & 15 & \\
\hline Total & 35 & 100 & 34 & 100 & \\
\hline \multicolumn{6}{|c|}{ Não ejacular dentro de seu parceiro previne a transmissão do HIV no sexo anal } \\
\hline \multicolumn{6}{|l|}{ Atitude de prevenção * } \\
\hline $\operatorname{Sim}$ & 15 & 43 & 31 & 91 & $<0,001$ \\
\hline Não & 20 & 57 & 3 & 9 & \\
\hline Total & 35 & 100 & 34 & 100 & \\
\hline \multicolumn{6}{|c|}{ No sexo oral, ter contato com o esperma não apresenta risco de infecção pelo HIV } \\
\hline \multicolumn{6}{|l|}{ Atitude de prevenção * } \\
\hline Sim & 15 & 43 & 31 & 9 & $<0,001$ \\
\hline Não & 20 & 57 & 3 & 9 & \\
\hline Total & 35 & 100 & 34 & 100 & \\
\hline
\end{tabular}

* Discorda (momento 1) Discorda (momento 2) e Concorda (momento 1) Discorda (momento 2);

** Concorda (momento 1) Concorda (momento 2) e Discorda (momento 1) Concorda (momento 2).

bre sexualidade e prevenção; articulado ao trabalho de intervenção dos coordenadores, possibilita transformá-las, partindo para a adoção de esquemas mais ajustados à realidade: prevenirse, pelo uso do preservativo ou de outras formas de prevenção, da infecção pelo HIV.

Quando HSH encontram-se numa situação de grupo, cuja proposta de discussão é abordar o tema "proteção e sexualidade", surge, nesse momento, segundo Fernandes 8, um princípio or- ganizador no qual se estabelece um processo de comunicação e de aprendizagem, pela troca de experiências, nas quais se expressam e transformam-se as representações que emergiram dessa interação. É nesse interjogo que se processa uma modificação interna (individual e grupal), a partir dos temas relacionados à proteção.

O estudo permite identificar que foi possível construir contextos mais amplos valendo-se da articulação de várias ECRO pessoais e grupais, a 
Distribuição dos participantes que permaneceram no estudo segundo características sócio-demográficas.

Início do estudo, momento anterior à intervenção.

\begin{tabular}{|c|c|c|c|c|c|c|c|}
\hline \multirow{2}{*}{ Característica } & \multicolumn{2}{|c|}{ Controle } & \multicolumn{2}{|c|}{ Intervenção } & \multicolumn{2}{|c|}{ Total } & \multirow[t]{2}{*}{$p$} \\
\hline & $\mathrm{n}$ & $\%$ & $n$ & $\%$ & $\mathrm{n}$ & $\%$ & \\
\hline \multicolumn{8}{|l|}{ Idade (anos) } \\
\hline $18-20$ & 2 & 5,0 & 3 & 9,0 & 5 & 7,0 & \\
\hline $20-40$ & 26 & 75,0 & 27 & 79,0 & 53 & 77,0 & \\
\hline $40-64$ & 7 & 20,0 & 4 & 12,0 & 11 & 16,0 & \\
\hline Total & 35 & 100,0 & 34 & 100,0 & 69 & 100,0 & \\
\hline Média & 30,3 & & 30,8 & & & & 0,842 * \\
\hline \multicolumn{8}{|l|}{ Local de nascimento } \\
\hline Norte/Nordeste & 7 & 20,0 & 6 & 18,0 & 13 & 19,0 & \\
\hline Centro-Oeste/Sudeste & 28 & 80,0 & 27 & 82,0 & 55 & 81,0 & \\
\hline Total & 35 & 100,0 & 33 & 100,0 & 68 & 100,0 & \\
\hline \multicolumn{8}{|l|}{ Tipo de moradia } \\
\hline Própria & 8 & 23,0 & 13 & 38,0 & 21 & 30,0 & \\
\hline Alugada & 13 & 37,0 & 9 & 27,0 & 22 & 32,0 & \\
\hline Parentes & 14 & 40,0 & 10 & 29,0 & 24 & 35,0 & \\
\hline Outro & 0 & 0,0 & 2 & 6,0 & 2 & 3,0 & \\
\hline Total & 35 & 100,0 & 34 & 100,0 & 69 & 100,0 & 0,206 ** \\
\hline \multicolumn{8}{|l|}{ Escolaridade (anos) } \\
\hline 8 e menos & 8 & 23,0 & 8 & 24,0 & 16 & 23,0 & \\
\hline Mais de 8 & 27 & 77,0 & 26 & 76,0 & 53 & 77,0 & \\
\hline Total & 35 & 100,0 & 34 & 100,0 & 69 & 100,0 & \\
\hline \multicolumn{8}{|c|}{ Renda per capita (salários mínimos: $R \$ 140,00$ ) } \\
\hline Até 1 & 5 & 16,0 & 5 & 19,0 & 10 & 17,0 & \\
\hline $1-2$ & 10 & 31,0 & 4 & 15,0 & 14 & 24,0 & \\
\hline $2 \vdash 5$ & 9 & 28,0 & 9 & 33,0 & 18 & 31,0 & \\
\hline $5-10$ & 7 & 22,0 & 6 & 22,0 & 13 & 22,0 & \\
\hline 10 e mais & 1 & 3,0 & 3 & 11,0 & 4 & 6,0 & \\
\hline Total & 32 & 100,0 & 27 & 100,0 & 59 & 100,0 & \\
\hline Mediana & 400 & & 575 & & & & 0,286 *** \\
\hline
\end{tabular}

* Teste t de Student;

** Teste de associação pelo qui-quadrado de Pearson;

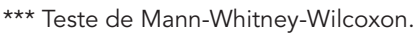

fim de compreenderem melhor como se protegerem do HIV. Isto se deu à medida que os participantes tomaram contato com contradições em seus discursos, nas polarizações da defesa de idéias, na superação de enrijecimentos e tensões que surgiram no processo grupal. Estes foram momentos do grupo promovidos pelo surgimento e articulação de questões (representações) tais como: identidade gay (revelar-se/ocultar-se), relacionamentos amorosos (abertos/fechados), representações sociais sobre o gay (preconceito/exaltação, o olhar da ciência), pacto conjugal (protetor/opressor), funções do gueto (exclusão/ proteção), diferenças e atritos na subcultura ho- mossexual (pobre/rico, festivo/discreto, jovens/ coroas).

Uma das representações surgidas no grupo ilustra, especialmente, como o acesso a conteúdos inconscientes pode ser transformado pelo processo grupal e ascender à consciência: tratase da representação sobre o preservativo como revelador de promiscuidade. A representação social do gay, trazida pelos participantes, está ligada à promiscuidade ("enquanto promíscuos, deveriam sempre usar preservativo”). Nesta equação, inconsciente, usar preservativo é assumir ser promíscuo, o que é inaceitável para muitas pessoas e grupos sociais e faz com que a informação 


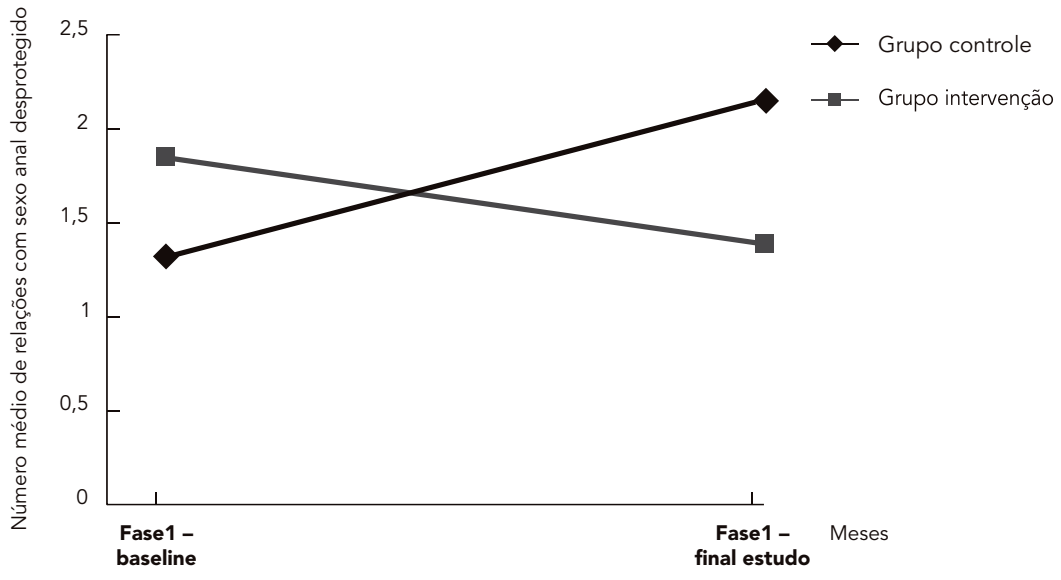

sobre prevenção via preservativo não funcione como medida de prevenção. Noutra situação, entre casais, a mesma representação impede a adoção de preservativo ("não sou promíscuo, preservativo não é necessário”). O trabalho sobre essa representação inconsciente, que se manifestou nas brechas dos discursos (contradições, lapsos, quebras etc.), possibilitou trazer à tona um novo sentido para a dificuldade em usar o preservativo e, provavelmente, promover-lhe o uso. Uma análise mais extensa dos conteúdos surgidos no trabalho com grupo operativo foi elaborada Colosio 18, com base em material advindo de transcrições das sessões de grupo.

É no tipo de trabalho descrito acima que grupo operativo diferencia-se, sem contrapor-se, de algumas abordagens preventivas, especialmente aquelas inspiradas em Paulo Freire. Apoiada no conceito de representação afetiva, tal como formulado por Freud, a relação afeto-razão trabalhada em grupo operativo distingue-se do que se entende, normalmente, como aspectos afetivos e racionais trabalhados em grupos (oficinas ou grupos de discussão) nos quais as pessoas debatem suas idéias e as emoções a elas ligadas, como nos grupos de Diaz 7, por exemplo. Enquanto procedimento de intervenção/investigação (pesquisa/ação), o grupo operativo fundamenta-se na psicologia social, que tem como objeto a relação entre fantasia inconsciente (representação) e estrutura social (posição social e papéis). Dessa forma, pretende capturar a dimensão inconsciente que atravessa e é atravessada pelo lugar ocupado socialmente pelas pessoas. Nessa medida, possibilita uma investigação e operação de natureza intrapsíquica, intersubjetiva e trans-subjetiva, que permite a transformação consciente e inconsciente das representações determinadas e mantidas pela história pessoal, pela condição social, econômica, política e cultural de cada sujeito e do grupo social. É por essa razão que, pela abordagem psicanalítica que fundamenta o grupo operativo, a transmissão de informações sobre como se proteger do HIV ou falar sobre afetos e emoções não é suficiente para articular e permitir uma efetiva mudança de comportamento.

Tome-se o trabalho de Diaz 7, que se baseou também numa intervenção em grupos. Embora sem alocação aleatória e grupo de controle, realizou-se em quatro sessões de grupos de discussão, coordenadas por dois facilitadores, homossexuais, latinos, especializados em saúde e orientados pelos princípios do "empowerment education", cujo pressuposto básico é não transmitir didaticamente informações ou recursos de prevenção. Na intervenção, procura estimular os participantes do grupo pela proposição de questões para reflexão crítica, dar retorno ao grupo sobre pontos de convergência e apresentação do que consideram como "fatos" sobre infecção HIV/AIDS (índices, pesquisas, documentos). Desse modo, privilegia a razão, reflexão e convencimento dos participantes via dados empíricos e conclusões lógicas sobre a melhor forma de se protegerem do HIV/AIDS. Em grupo operativo, não há proposi- 
ção de atividades, nem condução do trabalho do grupo pelos coordenadores, mas uma abordagem interpretativa dos conteúdos trazidos pelos participantes e dos mecanismos psíquicos que interatuam no momento da discussão, que possibilitam (ou impedem) a compreensão e a busca de sentidos para a não-prevenção.

O presente estudo, em concordância com Díaz 7 , identifica a necessidade de consideração de contextos mais amplos quando da elaboração de programas de prevenção, para que não se restrinjam ao treinamento do uso do preservativo, em recomendações médicas e dados estatísticos. Ainda, que superem abordagens comportamentais ou pedagógicas, as quais concebem o usuário como mero receptor de informações e que, segundo Paiva 4 , continuam a orientar a maior parte dos trabalhos em prevenção nos serviços públicos de saúde.

Por todas essas características, o grupo operativo permite lidar com as dimensões da vulnerabilidade, tal como definida por Ayres et al. 19, à medida que os temas das discussões acerca de uma série de representações sobre a homossexualidade e a prevenção de HIV surgem, em grande parte, na forma de opiniões polarizadas e enrijecidas. Esses temas coincidem com aqueles que têm sido apontados por diversos pesquisadores de prevenção entre HSH 4,5,6,7,12 como fundamentais para um efetivo trabalho de mudança. São eles: a questão da identidade homossexual, da revelação da identidade/intimidade ao grupo familiar e/ou social, relacionamentos amorosos e fidelidade, padrões de comportamento (discreto/festivo, ativo/passivo, afeminado/masculino, promiscuidade/estabilidade etc.).

As questões metodológicas que se apresentaram neste estudo foram de grande importância, a começar pela dificuldade de diálogo entre diversificadas abordagens encontradas, especialmente na área da psicologia. Esta é uma questão importante na produção científica internacional em prevenção, como aponta recente revisão bibliográfica dos estudos de intervenção dirigidos à prevenção de HIV entre HSH realizada por Johnson et al. ${ }^{12}$, a qual revela que poucos estudos apresentam desenhos padronizados, com controle rigoroso de resultados e tratamento estatístico adequado. Aponta como necessária a realização de pesquisas que utilizem estratégias preventivas semelhantes à utilizada neste estudo, abordando questões de validade e confiabilidade da mensuração de questões de comportamento sexual auto-reportado, como discutido por Catania et al. 15. Uma outra questão referese à identificação de limitações metodológicas neste estudo quanto à possibilidade de viés de cortesia e de seguimento, que foi parcialmente controlado pela aleatorização e que teve reflexo nos percentuais semelhantes de perdas nos dois grupos. E também quanto à baixa validade externa dos achados, uma vez que os participantes são usuários e voluntários de pesquisa provenientes de serviços da rede municipal de saúde de São Paulo.

Quanto aos resultados obtidos, a concordância entre as respostas de questões sobre crenças, conhecimentos sobre HIV/AIDS (Tabela 2) e queda do número médio de ato sexual desprotegido no grupo intervenção (Figura 2) reforça o efeito favorável da intervenção a favor da prevenção, apesar de não garantir mudanças efetivas de comportamento. Um efeito possível do grupo operativo revela-se, ao avaliarem-se as respostas do questionário sobre crenças, atitudes e conhecimentos, na verificação de uma modificação sensível no nível das respostas, na mesma direção apontada acima, em favor de uma prática de sexo mais seguro. Entre as questões analisadas, uma mostrou-se contraditória em relação às outras: a afirmação de que "repetidas exposições ao HIV, com o tempo, podem prejudicar o sistema imunológico do portador". Entende-se que, por tratar-se de um conhecimento mais refinado dos mecanismos de infecção, associado ao fato de ter sido apresentada em ordem inversa às demais, pode ter causado dificuldades de entendimento aos participantes.

A estratégia de avaliar o impacto de intervenção pela análise do aumento do uso de preservativo deve-se também ao fato de que a adoção desse item foi uma das diretrizes estratégicas adotadas no Brasil e no mundo, desde o começo da epidemia, especialmente para a comunidade homossexual. Alguns estudos, como os de Paiva et al. 20 e Hearst \& Chen 21, têm sido elaborados com o propósito de desenvolver uma tecnologia de prevenção que difunda e sistematize seu uso e, por isso, a importância de conhecer melhor a prática sexual de alguns grupos. Os autores apontam mudança significativa na forma como a promoção do uso de preservativo dirigida à população em geral vem se transformando ao longo do tempo, assim como destacam a importância de que, ao lado da abundância e abrangência das possibilidades de aquisição de preservativo, o processo de sua promoção seja acompanhado de estratégias que se integrem, a fim de promovêlo como a forma mais eficiente, barata e fácil na prevenção do HIV.

Finalizando, além das dificuldades que a área de prevenção vem enfrentando no trabalho de prevenção do HIV, especialmente entre jovens, novos desafios têm sido lançados como o fenômeno do "barebacking", já não tão incipiente como há alguns anos, segundo Halkitis et al. 22. 
Solicita-se, com urgência, da área de prevenção uma compreensão mais profunda, integrada e multidisciplinar dos fatores psicossociais envolvidos na sua emergência; nesse sentido, devem considerar as várias esferas (política, econômica, social, do imaginário, do simbólico e do subjetivo - em sua dimensão consciente e inconsciente) que se organizam, interatuam e concorrem na vida cotidiana das pessoas e de seus grupos sociais. Assim, não só o grupo operativo, como também os outros procedimentos que propõem esse tipo de contextualização poderiam ser considerados com maior amplitude nos programas e pesquisas de prevenção de HIV/AIDS.

\section{Resumo}

Este estudo focaliza o grupo operativo como intervenção preventiva de HIV para HSH (homens que fazem sexo com homens), usuários de serviços de saúde pública de São Paulo, Brasil. Foram distribuídos, por sorteio aleatório, 100 voluntários em dois grupos (intervenção e controle - 50 cada um). Todos eles responderam a questionários em duas fases distintas: antes da intervenção e seis meses depois de serem submetidos a ela. A avaliação do efeito da intervenção foi obtida pela variação do número médio de relações sexuais anais sem preservativo e análise de respostas sobre infecção pelo HIV. Terminaram o estudo 69 participantes (34 grupo de intervenção; 35 - grupo de controle). No grupo de intervenção, observou-se, pelos dados obtidos, uma diminuição da prática de sexo anal desprotegida $(p=0,029)$ e aumento do número médio de respostas favoráveis à prevenção. Esses índices indicam ser a população estudada sensível à mudança em favor da adoção da prática de sexo mais seguro mediante participação no grupo operativo. Novos estudos são necessários para avaliar a possibilidade de utilização dessa abordagem nos serviços públicos de saúde e para outras populações.

HIV; Sindrome de Imunodeficiência Adquirida; Homossexualidade Masculina; Prevenção de Doenças; Psicologia Social

\section{Contribuições}

R. Colosio participou na intervenção, pesquisa de campo, coleta e análise de dados, elaboração do artigo. M. I. A. Fernandes participou das sessões de grupo operativo e participou da análise qualitativa das transcrições e elaboração do artigo. D. P. Bergamaschi foi responsável pela assessoria em estatística e participou da elaboração da estratégia de coleta e análise de dados quantitativos, como também da elaboração do artigo. I. R. Scarcelli participou das sessões de grupo operativo e da elaboração do artigo. I. C. Lopes participou das sessões de grupo operativo. N. Hearst participou da análise estatística dos dados quantitativos e da elaboração do artigo.

\section{Agradecimentos}

Área Temática DST/AIDS da Coordenação da Gestão Descentralizada da Secretaria Municipal da Saúde de São Paulo, em especial aos profissionais de saúde dos CTA Henfil e Santo Amaro e ao Centro de Referência e Treinamento do Programa Estadual de DST/AIDS de São Paulo. Ao Center for AIDS Prevention Studies, University of California-San Francisco, pelo apoio financeiro e formação em metodologia de pesquisa em AIDS. 


\section{Referências}

1. Ministério da Saúde. Boletim Epidemiológico de AIDS 2002; Ano XVI; no. 1.

2. Secretaria Municipal de Saúde de São Paulo. Boletim Epidemiológico de AIDS 2002; Ano VI, no. 6.

3. Veras MASM, Bergamaschi DP, Mesquita F, Paneque NA, Carvalho SV, Zular OS, et al. Infecção pelo HIV em usuário dos Centros de Testagem e Aconselhamento (CTA), da rede municipal de saúde de São Paulo, 2000-2001: diferenças entre os gêneros. Boletim Epidemiológico de AIDS do Município de São Paulo 2002; 6:29-30.

4. Paiva V. Sem mágicas soluções: a prevenção e o cuidado em HIV/AIDS e o processo de emancipação psicossocial. Interface Comum Saúde Educ 2002; 6:25-38.

5. Parker R. Na contramão da AIDS: sexualidade, intervenção, política. Rio de Janeiro: Associação Brasileira Interdisciplinar de AIDS/Editora 34; 2000.

6. Terto Jr. V. La salud de los hombres gay y otros hombres que tienen sexo con hombres: desafíos para la tercera década de la epidemia de VIH/SIDA. In: Carceres CF, Pecheny M, Terto Jr. V, editores. SIDA y sexo entre hombres en América Latina: vulnerabilidades, fortalezas y propuestas para la acción. Perspectivas y reflexiones desde la salud pública, las ciencias sociales y el activismo. Lima: Universidad Peruana Cayetano Heredia/Programa Conjunto de Naciones Unidas para el SIDA; 2002. p. 167-77.

7. Díaz RM. Latino gay man and HIV: culture, sexuality and risk behavior. New York: Routledge; 1998.

8. Fernandes MIA. De como emerge a questão do narcisismo e da alteridade no grupo operativo [Tese de Doutorado]. São Paulo: Instituto de Psicologia, Universidade de São Paulo; 1989.

9. Freud S. Uma nota sobre o inconsciente na psicanálise. In: Obras completas. v. XII. Rio de Janeiro: Editora Imago; 1996. p. 275-85.

10. Pichon-Rivière E. Teoria do vínculo. São Paulo: Editora Martins Fontes; 1982.

11. Pichon-Rivière E. O processo grupal. São Paulo: Editora Martins Fontes; 1988.
12. Johnson WD, Hedges LV, Diaz RM. Interventions to modify sexual risk behaviors for preventing HIV infection in men who have sex with men (Cochrane Review). In: The Cochrane Library, Issue 1, 2004. Oxford: Update Software.

13. Moreno DMFC. A saúde pública e a psicanálise: a produção do conhecimento no Brasil acerca da AIDS [Dissertação de Mestrado]. São Paulo: Faculdade de Saúde Pública, Universidade de São Paulo; 2001.

14. Hulley SB, Cummings SR, editors. Designing clinical research: an epidemiologic approach. Baltimore: Williams \& Wilkins; 1988.

15. Catania JA, Kegeles SM, Coates TJ. Towards and understanding of risk behavior: an AIDS Risk Reduction Model (ARRM). Health Educ Q 1990; 17:53-72.

16. Nunnally JC, Bernstein IH. Psychometric theory. 3rd Ed. New York: MacGraw-Hill; 1994.

17. Vittnghoff E, Douglas J, Judson F, McKirnan D, MacQueen K, Buchbinder SP. Per-contact risk of human immunodeficiency virus transmission between male sexual partners. Am J Epidemiol 1999; 150:306-11.

18. Colosio R. O grupo operativo como instrumento de intervenção na área da saúde: um estudo em prevenção de DST/AIDS na cidade de São Paulo [Dissertação de Mestrado]. São Paulo: Instituto de Psicologia, Universidade de São Paulo; 2005.

19. Ayres JRCM, França-Junior I, Calazans GJ, SalettiFilho HC. O conceito de vulnerabilidade e as práticas de saúde: novas perspectivas e desafios. In: Czeresnia D, organizadora. Promoção da saúde: conceitos, reflexões, tendências. Rio de Janeiro: Editora Fiocruz; 2003. p. 117-39.

20. Paiva V, Venturi G, França-Junior I, Lopes F. Uso de preservativos - Pesquisa Nacional MS/IBOPE 2003. http://www.aids.gov.br (acessado em 12/ Fev/2004).

21. Hearst N, Chen S. Condom promotion for AIDS prevention in the developing world: is it working? Stud Fam Plann 2004; 35:39-47.

22. Halkitis PN, Parsons JT, Wilton L. Barebacking among gay and bissexual men in New York City: explanations for the emergence of intentional unsafe behavior. Arch Sex Behav 2003; 32:351-7.

Recebido em 16/Jan/2006

Versão final reapresentada em 07/Ago/2006

Aprovado em 18/Ago/2006 